\title{
Clinical validation of an audio-based uroflowmetry app in adult males
}

Mark T. Dawidek, MD, MSc ${ }^{1}$; Rohit Singla, MASc, $\mathrm{MD} / \mathrm{PhD}[\mathrm{c}]^{2}$; Lucie Spooner, $\mathrm{MBChB}$, $\mathrm{MRCS}^{1}$; Louisa Ho, MD ${ }^{1}$; Christopher Nguan, MD, FRCSC ${ }^{1,2}$

${ }^{1}$ Department of Urologic Sciences, University of British Columbia, Vancouver, BC, Canada;

${ }^{2}$ Faculty of Medicine, University of British Columbia, Vancouver, BC, Canada

Acknowledgments: This study was supported by the CUA-Pfizer Urology Resident Grant Program. The authors would also like to thank John Brohan of Traders Micro, Montreal, QC, Canada for technical support.

Cite as: Dawidek MT, Singla R, Spooner L, et al. Clinical validation of an audio-based uroflowmetry app in adult males. Can Urol Assoc J 2021 October 18; Epub ahead of print. http://dx.doi.org/10.5489/cuaj.7362

Published online October 18, 2021

Corresponding author: Mark T. Dawidek, Department of Urologic Sciences, University of British Columbia, Vancouver, BC, Canada; mark.dawidek@gmail.com

$* * *$

\section{Abstract}

Introduction: Uroflowmetry is a common test to evaluate lower urinary tract symptoms. Audiobased uroflowmetry is a novel, alternative approach that determines urine flow by measuring sound. Available as a smartphone application, it has potential for screening and monitoring common urological pathologies, particularly in out-of-office environments. This study is the first to evaluate audio-based uroflowmetry in a clinical setting against the gold standard.

Methods: Adult male patients $(\mathrm{n}=44)$ attending a general urology clinic were recruited. Audiobased uroflowmetry and conventional uroflowmetry were performed concurrently. Pearson correlation and Bland-Altman analysis were used to compare performance with respect to max flow, time to max flow, and total voiding time. Symmetric mean absolute percentage error (SMAPE) was used to compare curve shapes. Repeatability was evaluated separately in three healthy volunteers using repeat measures correlation.

Results: Among urology clinic patients, the correlation for max flow was 0.12. Correlation for time to max flow was 0.46 , with limits of agreement of $-120-165 \%$. Correlation for total voiding time was 0.91 , with limits of agreement of $-41-38 \%$. The SMAPE for curve shape was $32.6 \%$, with corresponding accuracy of $67.4 \%$. Among healthy volunteers, the repeat measures correlation for max flow was 0.72 . 
Conclusions: Audio-based uroflowmetry was inconsistent in evaluating flow rate, attributable to high variability and difficult standardization for acoustic signals. Performance improved with respect to temporal variables, as well as flow curve shape. Further work evaluating intra-patient reliability and pathology-specific performance is required to fully evaluate audio-based uroflowmetry as a screening or monitoring tool.

\section{Introduction:}

Lower urinary tract symptoms (LUTS) significantly impact approximately half of men and women aged $>40$ years, with medical morbidity, quality of life, and healthcare cost implications. ${ }^{1}$ The presentation and etiology for LUTS tend to differ between men and women, with bladder outlet obstruction (BOO) and benign prostatic hyperplasia (BPH) being the most common presentation and etiology respectively amongst men. ${ }^{2}$ Uroflowmetry is a wellestablished clinic-based test to evaluate LUTS, used predominantly in the context of BOO and $\mathrm{BPH}^{3-6}$ Conventionally, a weight transducer is used to measure urine volume voided per unit time and generate a flow curve. This provides flow pattern, maximum flow rate $\left(\mathrm{Q}_{\max }\right)$, time to maximum $\left(\mathrm{T}_{\max }\right)$, total voiding time $\left(\mathrm{T}_{\mathrm{tot}}\right)$, and total voided volume $\left(\mathrm{V}_{\text {tot }}\right)$ for clinical interpretation (Figure 1). ${ }^{4}$

There is known incongruence between clinic-based versus home-based voiding measurement, attributable to the artificial circumstances of voiding in clinic (i.e. pre-hydration, voiding on demand, decreased privacy). ${ }^{7}$ Furthermore, measuring multiple sequential voids might decrease variability and yield more accurate results, but is logistically impractical in clinic. ${ }^{7}$ This has prompted the development of home-based uroflowmetry devices. ${ }^{8}$ However, these devices either provide limited measurements, are complicated to use, or are prohibitively expensive, and none have been widely adopted. ${ }^{7}$ There remains a need for a low-cost, convenient, home-based method for routine evaluation of LUTS.

One promising alternative is the use of sound. Audio-based uroflowmetry uses the sound of a patient voiding to generate a flow curve and derive the same parameters as conventional uroflowmetry. Such audio-based uroflowmetry can be made available as a software application on any modern mobile device with a microphone, making it low cost, simple, and portable. This paradigm has potential for screening and monitoring common urologic pathologies, particularly in out-of-office environments. However, to date, it has not been robustly evaluated in a clinical setting against the gold standard. ${ }^{9,10}$ This study compares the accuracy of an audio-based uroflowmetry application against conventional uroflowmetry in adult male patients presenting to the urology clinic.

\section{Methods}




\section{Study population}

There were two separate cohorts of study participants. First, a cohort of adult (age $>18$ years) male patients attending a general urology clinic at Vancouver General Hospital (Vancouver, BC, Canada) was recruited for the purpose of evaluating audio-based uroflowmetry against conventional uroflowmetry in a clinical setting. This demographic was selected based on the high prevalence of BOO. Furthermore, previous studies demonstrated superior performance of audio-based uroflowmetry within this group. ${ }^{9}$ Patients were recruited sequentially as they presented to urology clinic, after uroflowmetry had been ordered based on the clinical judgement of a urologist. There were no specific urologic history or diagnosis inclusion criteria. Patient charts were subsequently reviewed to collect demographic and clinical information, including age, reason for visit, and lower urinary tract diagnoses. Second, a cohort of healthy adult male volunteers with no urologic medical history was recruited for the purpose of validating intraindividual repeatability. The Clinical Research Ethics Board of the University of British Columbia approved this study (H18-00188) and written informed consent was obtained from all participants.

\section{Data collection}

Conventional uroflowmetry was performed using the Urocap IV device (Laborie, Brossard, QC, Canada). A manufacturer-provided software module was used for digital export of raw data. The Urocap IV device includes an optional funnel to assist participants in performing the test but is not required to obtain measurements. The funnel unpredictably alters the acoustics of voiding and was therefore replaced with a metal receptacle which more closely simulates a conventional toilet.

Audio-based uroflowmetry was performed using the publicly available TeleSonoUroFlow application (Traders Micro, Montreal, QC, Canada). The application generates a flow curve from the acoustic intensity of urine striking a surface. A concurrent audio recording of the void is also obtained. A modified version of the application, through a collaboration with the original developer, enabled digital export of raw data. The application is compatible with the built-in microphone on any Android or iOS mobile device. Measurements for this study were made using the same BLU R1 HD device (BLU, Miami, FL, USA). The mobile device was set-up with consistent height and orientation beside the Laborie device.

Conventional and audio-based uroflowmetry were performed simultaneously for each void (Figure 2). All set-up was performed by research staff and participants were not required to interact with devices in any manner. The urology clinic patients provided a single void, while the healthy volunteers provided repeated voids on separate occasions. Data collection was otherwise identical for both cohorts.

\section{Data analysis}


Audio recordings were reviewed by a blinded individual to ensure adequate quality and to isolate the trial interval. All artefacts from background noise were retained. Participants with $\mathrm{V}_{\text {tot }}$ $<150 \mathrm{cc}$ were excluded. Data from both conventional and audio-based uroflowmetry was exported into MATLAB (MathWorks, Natick, MA, USA), where algorithms were developed to automate and ensure reliability of comparisons.

For each void, the dominant curves were first isolated (i.e. small, late dribbles were excluded). The parameters $\mathrm{Q}_{\max }, \mathrm{T}_{\max }$, and $\mathrm{T}_{\text {tot }}$ were then independently extracted for the conventional and audio-based flow curves. The amplitudes of the corresponding curves were then linearly normalized using $\mathrm{Q}_{\max }$ and the curves were temporally aligned using a built-in MATLAB function. Lastly, the symmetric mean absolute percentage error (SMAPE) between the two curves was calculated according to (1), where $\mathrm{Q}_{\text {conventional }}$ and $\mathrm{Q}_{\text {audio }}$ are the flow rates for conventional and audio-based uroflowmetry at each timepoint.

$$
S M A P E=\frac{100 \%}{n} \sum_{t=T_{\text {tot }}}^{n} \frac{\left|Q_{\text {conventional }}-Q_{\text {audio }}\right|}{Q_{\text {conventional }}+Q_{\text {audio }}}
$$

The outcomes measured in this study were uroflowmetry parameters $Q_{\max }, T_{\max }$, and $T_{\text {tot }}$ as well as flow curve shape. Pearson correlation coefficient was used to compare $\mathrm{Q}_{\max }, \mathrm{T}_{\max }$, and $\mathrm{T}_{\text {tot. }}$ Bland-Altman analysis was additionally used to evaluate agreement between $\mathrm{T}_{\max }$ and $\mathrm{T}_{\text {tot. }}{ }^{11}$ The limits of agreement (LoA) were defined by a $95 \%$ confidence interval. Bland-Altman analysis could not be used to compare $\mathrm{Q}_{\max }$ due to discrepant units of measure. SMAPE was used to quantitively compare the shapes of corresponding flow curves. ${ }^{12}$ The calculation of SMAPE in (1) ensures a percentage error between 0 to $100 \%$, and accuracy is subsequently obtained by subtracting percentage error from $100 \%$. This method was selected due to the intuitive interpretation of accuracy from a percentage. Repeated measures correlation was used to evaluate the repeatability of $\mathrm{Q}_{\max }$ across multiple voids in the healthy volunteers. ${ }^{13}$

\section{Results}

A total of 94 voids were obtained from patients attending urology clinic (single void per individual). Final analysis included 44 voids -22 were excluded for $\mathrm{V}_{\text {tot }}<150 \mathrm{cc}$ and 28 were excluded for technical issues (12 unusable Urocap IV datafiles, 9 unusable TeleSonoUroFlow datafiles, 5 activation/time-out issues, 2 severe background noise). Demographic and clinical information for the patients is provided in Table 1. A further 25 voids were obtained from three healthy volunteers (5-10 repeated voids per individual). Technical issues occurred completely at random and affected healthy volunteers at a similar rate as clinic patients.

For patients attending urology clinic, the $\mathrm{Q}_{\max }$ Pearson correlation between conventional and audio-based uroflowmetry was $0.12(p=0.42)$ (Figure 3$)$. The $\mathrm{T}_{\max }$ correlation was $0.46(p<$ 0.05 ), with Bland-Altman LoA of -120 to $165 \%$ (Figure 4$)$. The $\mathrm{T}_{\text {tot }}$ correlation was 0.91 ( $p<$ 0.05 ) with LoA of -41 to $38 \%$ (Figure 4). The SMAPE between audio-based and conventional 
uroflowmetry curve shapes ranged from 11.5 to $71.2 \%$, with a mean of $32.6 \%$ (SD 16\%) corresponding to an accuracy of $67.4 \%$ (Figure 5).

For healthy volunteers, the $\mathrm{Q}_{\max }$ repeated measures correlation between conventional and audio-based uroflowmetry was $0.72(p<0.05)$ (Figure 3$)$. The SMAPE for curve shape was $17.7 \%$ (SD $6.6 \%$ ), corresponding to an accuracy of $82.3 \%$.

\section{Discussion}

Audio-based uroflowmetry was inconsistent in evaluating maximum flow rate in patients attending urology clinic when compared to conventional uroflowmetry, reflected by the $\mathrm{Q}_{\max }$ correlation of 0.12 . This is attributable to the high variability and difficult standardization of sound signals. While our set-up accounted for these factors as best as possible, audio-based measurement of $Q_{\max }$ is highly sensitive to height of stream, angle of stream, receptacle properties, microphone position, and room acoustics. ${ }^{9,10}$ In conventional uroflowmetry $10-15 \mathrm{cc} / \mathrm{s}$ is an established cut-off indicating BOO; based on our results, it is not possible to establish a universal audio-based $\mathrm{Q}_{\max }$ cut-off which differentiates normal from abnormal voiding. ${ }^{6}$ Interestingly, the $\mathrm{Q}_{\max }$ correlation improved among the healthy volunteers. While our patient data consisted of single measurements per individual, our healthy volunteer data involved multiple measurements per individual. The repeated measures correlation of 0.72 is likely attributable to high intra-individual reliability, which is supported by previous reports of high test-to-test reproducibility in audio-based uroflowmetry. ${ }^{14,15}$ This would suggest that despite it being infeasible to establish a universal audio-based $\mathrm{Q}_{\max }$ cut-off, it might be possible to define normal values and detect changes in voiding on an individual basis. This study was not designed to evaluate intra-individual reliability in patients, as it is challenging to obtain repeat measures when individuals present to clinic a few times per year, but this certainly warrants further investigation.

Audio-based uroflowmetry performance improved with respect to temporal variables, with $\mathrm{T}_{\max }$ correlation of 0.46 and $\mathrm{T}_{\text {tot }}$ correlation of 0.91 . The Bland-Altman plot for $\mathrm{T}_{\max }$ showed a mean difference of measures of $28 \%$, suggesting a bias toward earlier $\mathrm{T}_{\max }$ when measured using audio-base uroflowmetry. This is likely an artefact of the urine stream's impulse noise as it first strikes the water surface, resulting in an earlier peak signal amplitude. The Bland-Altman plot for $\mathrm{T}_{\text {tot }}$ showed minimal bias. Indeed, with LoA of -41 to $38 \%, \mathrm{~T}_{\text {tot }}$ performance was particularly robust. This is likely because establishing the start and end of voiding is a binary condition, clearly defined by presence versus absence of either flow or sound. The discrepant performance of audio-based uroflowmetry between flow and temporal measures is consistent with previous investigations, with reports of $\mathrm{Q}_{\max }$ correlation of 0.38 versus $\mathrm{T}_{\text {tot }}$ correlation of 0.87 to $0.95 .9,10$

Flow curve pattern is important subjective information garnered from uroflowmetry and is reliant on the objective accuracy of the curve shape. Previous investigations have not compared curve shape between audio-based and conventional uroflowmetry. ${ }^{9,10,14,1516,17} \mathrm{We}$ 
report an accuracy of $67.4 \%$ in patients and $82.3 \%$ in healthy individuals, suggesting audio-based uroflowmetry yields reasonable representation of flow curve amenable to further analysis of pattern.

The most significant limitation in our study was a high exclusion rate due to technical issues (28 of 94 patients). In such cases, either the Urocap IV device or the TeleSonoUroFlow application was improperly activated, timed-out before the patient voided, or yielded corrupted raw digital data. However, this simultaneously emphasizes a potential advantage of simple, home-based methods such as audio-based uroflowmetry. When performing uroflowmetry in clinic, time constraints make it is logistically impractical to repeat a measurement once a patient has voided should technical issues arise. Audio-based uroflowmetry could potentially lower the barriers to obtaining repeat measurements to account for technical mishaps and unrepresentative or outlier voids. This requires further assessment of audio-based uroflowmetry performance in the home environment.

The potential to generate frequent out-of-office datapoints has other important implications. There is known incongruence between voiding measured in clinic versus measured at home. ${ }^{7}$ In particular, there is a phenomenon of increasing $Q_{\max }$ with successive clinic visits simply from familiarisation with the uroflowmetry test. ${ }^{18}$ Furthermore, studies have demonstrated inherent variability, especially circadian fluctuation, in voiding parameters. ${ }^{16}$ Recent evaluation of over 19,000 voids in over 600 patients using home-base uroflowmetry suggested a coefficient of variation of $27.6 \%$ for $\mathrm{Q}_{\max .}{ }^{19}$ Others have highlighted that this variability is greatest in patients with voiding pathology rather than in healthy controls. ${ }^{20}$ There is significant information lost to the urologist evaluating LUTS using sparse, clinic-based conventional uroflowmetry which might be better captured using frequent, out-of-office audiobased uroflowmetry.

To our knowledge, this is the first study to evaluate audio-based uroflowmetry in a clinical setting. While our results suggest it is not equivalent to conventional uroflowmetry, it does provide reasonable assessment of temporal voiding parameters and representation of flow curve shape. Taken in conjunction with its simplicity and convenience, enabling the generation of abundant out-of-office datapoints, audio-based uroflowmetry has potential as a screening and monitoring tool for voiding pathology. While this study included all-comer male urology clinic patients, future work should focus on the detection and evaluation of specific pathologies. Audio-based uroflowmetry is particularly promising as a tool for monitoring progression, response to treatment, and recurrence. To this end, future work should investigate the intrapatient reliability of audio-based uroflowmetry and its ability to detect longitudinal changes in voiding.

\section{Conclusions}

Audio-based uroflowmetry is not an equivalent replacement for conventional uroflowmetry. However, it merits further study in the home environment to establish whether it can reliably 
detect abnormalities or changes in voiding. It provides a sufficient representation of voiding that it has potential as a screening or monitoring tool which would prompt further evaluation such as conventional uroflowmetry. 


\section{References}

1. Coyne KS, Sexton CC, Thompson CL, et al. The prevalence of lower urinary tract symptoms (LUTS) in the USA, the UK and Sweden: Results from the epidemiology of LUTS (EpiLUTS) study. BJU Int 2009;10:352-60.

2. Parsons JK. Benign prostatic hyperplasia and male lower urinary tract symptoms: Epidemiology and risk factors. Curr Bladder Dysfunct Rep 2010;5:212-18.

3. Reynard JM, Yang Q, Donovan JL, et al. The ICS-'BPH' Study: Uroflowmetry, lower urinary tract symptoms and bladder outlet obstruction. Br J Urol 1998;82:619-23.

4. Kelly CE. Evaluation of voiding dysfunction and measurement of bladder volume. Rev Urol 2004;6:S32-37.

5. Singla S, Garg R, Singla A, et al. Experience with uroflowmetry in evaluation of lower urinary tract symptoms in patients with benign prostatic hyperplasia. J Clin Diagn Res 2014;8:1-3.

6. Oelke M, Höfner K, Jonas U, et al. Diagnostic accuracy of noninvasive tests to evaluate bladder outlet obstruction in men: Detrusor wall thickness, uroflowmetry, postvoid residual urine, and prostate volume. Eur Urol 2007;52:827-35.

7. Bray A, Griffiths C, Drinnan M, et al. Methods and value of home uroflowmetry in the assessment of men with lower urinary tract symptoms: A literature review. Neurourol Urodyn 2012;31:7-12.

8. Chun K, Kim SJ, Cho ST. Noninvasive medical tools for evaluating voiding pattern in real life. Int Neurourol J 2017;21:10-16.

9. Krhut J, Gärtner M, Sýkora R, et al. Comparison between uroflowmetry and sonouroflowmetry in recording of urinary flow in healthy men. Int J Urol 2015;22:76165.

10. Gartner M, Krhut J, Hurtik P, et al. Evaluation of voiding parameters in healthy women using sound analysis. Low Urin Tract Symptoms 2018;10:12-16.

11. Martin Bland J, Altman DG. Statistical methods for assessing agreement between two methods of clinical measurements. Lancet. 1986;327:307-10.

12. Flores BE. A pragmatic view of accuracy measurement in forecasting. Omega. 1986;14:93-98.

13. Bakdash JZ, Marusich LR. Repeated measures correlation. Front Psychol 2017;8:456.

14. Zvarova K, Ursiny M, Giebink T, et al. Recording urinary flow and lower urinary tract symptoms using sonouroflowmetry. Can J Urol. 2011;18:5689-94.

15. Zvara P, Ursiny M, Giebink T, et al. Comparison of test-to-test variability in flow parameters recorded with a wireless-based acoustic system with that of standard uroflowmetry. Int Urogynecol J 2011;22:S1 105-06.

16. Porru D, Scarpa RM, Prezioso D, et al. Home and office uroflowmetry for evaluation of LUTS from benign prostatic enlargement. Prostate Cancer Prostatic Dis 2005;8:45-49.

17. Sonke GS, Kiemeney LA, Verbeek AL, et al. Low reproducibility of maximum urinary flow rate determined by portable flowmetry. Neurourol Urodyn 1999;18:183-91.

18. Reynard JM, Peters TJ, Lim C, et al. The value of multiple free-flow studies in men with lower urinary tract symptoms. Brit J Urol 1996;77:813-18.

19. Summers SJ, Armstrong JM, Kaplan SA, et al. Male voiding behavior: Insight from 19,824 at-home uroflow profiles. J Urol 2021;205:1126-32. 
20. Golomb J, Lindner A, Siegel Y, et al. Variability and circadian changes in home uroflowmetry in patients with benign prostatic hyperplasia compared to normal controls. J Urol 1992;147:1044-47.

Figures and Tables

Fig. 1. Uroflowmetry curve.

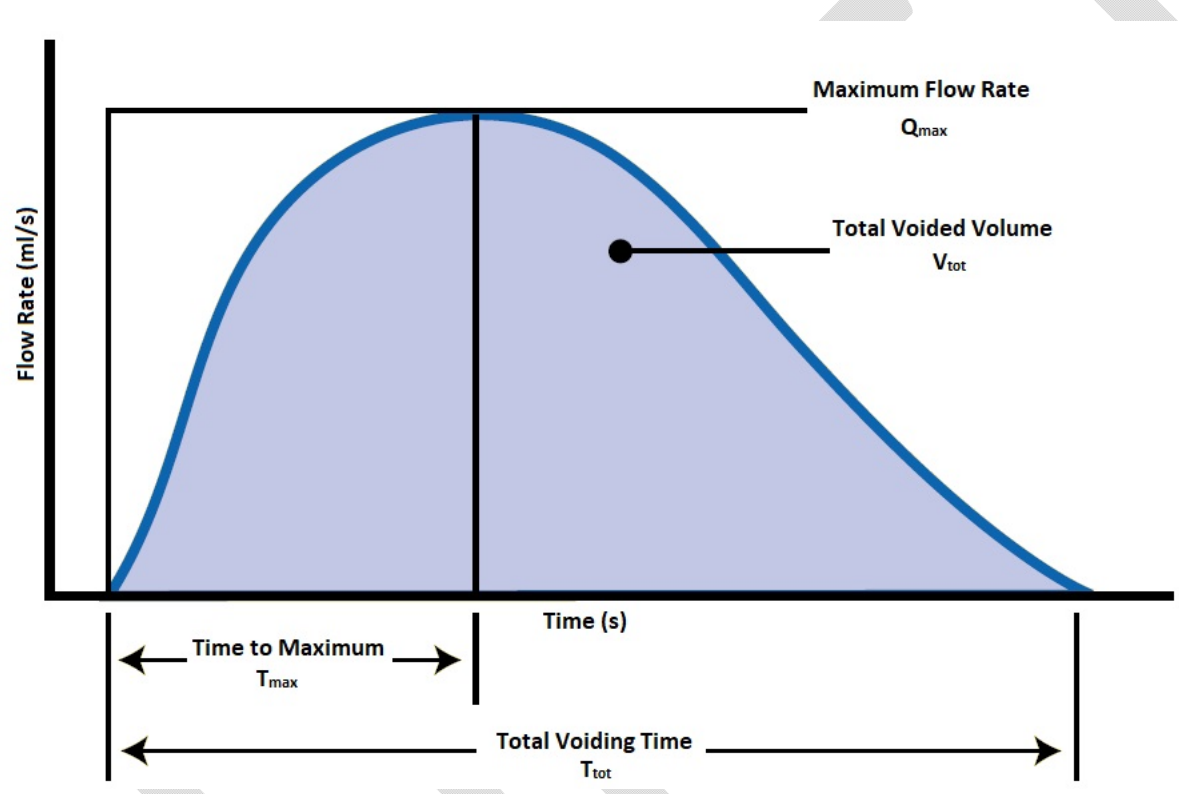


Fig. 2. Illustration of trial setup (a) individuals stand to void and conventional and audio-based uroflowmetry are performed simultaneously (b) usual funnel used with Laborie Urocap IV device is replaced with a metal receptacle partially filled with water $(\boldsymbol{c})$ the mobile device with the TeleSonoUroflow app is positioned at standardized height and mic orientation.

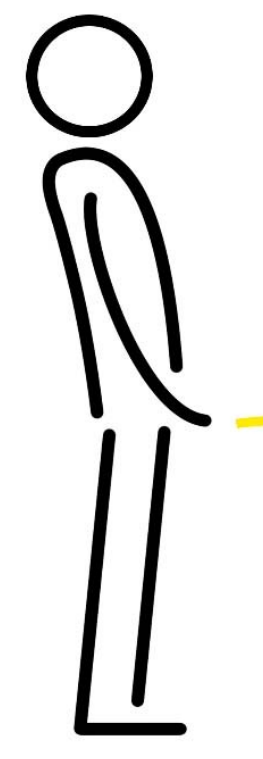

a

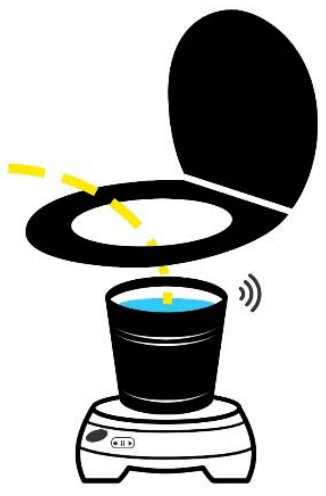

b

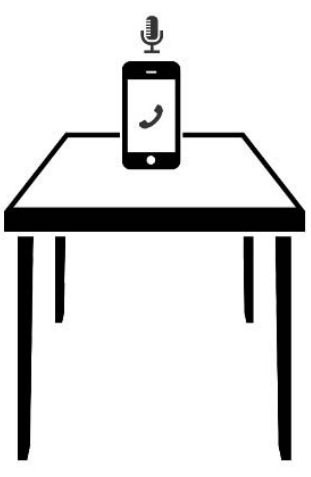

C

Fig. 3. $Q_{\max }$ in conventional versus audio-based uroflowmetry (A) using Pearson correlation in patients attending urology clinic and $(\boldsymbol{B})$ using repeat measures correlation in healthy individuals (participants plotted individually).

A
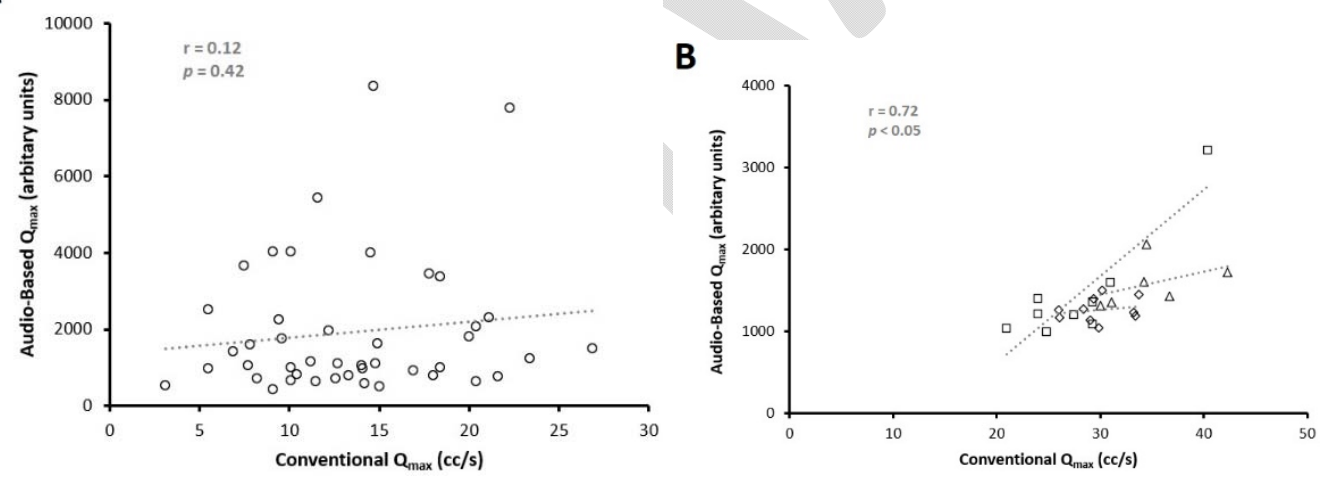
Fig. 4. Bland-Altman analysis between conventional and audio-based uroflowmetry in patients attending urology clinic for (A) $\mathrm{T}_{\max }$ and (B) $\mathrm{T}_{\text {tot. }}$

A

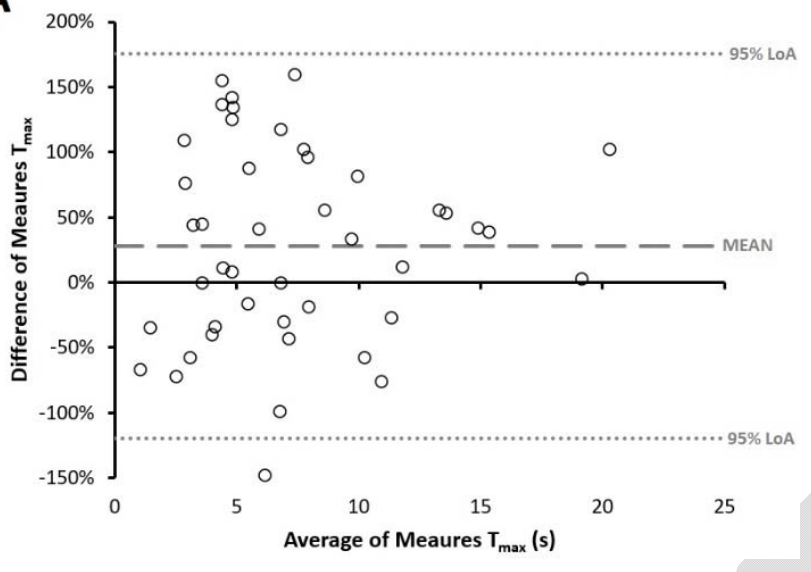

B

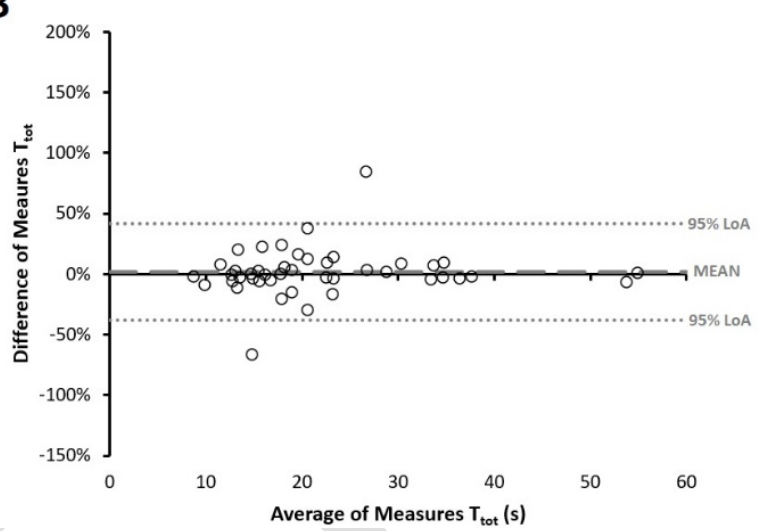

Fig. 5. Representative sample of flow curve shapes measured using audio-based and conventional uroflowmetry in three patients attending urology clinic. SMAPE ranged from 11.5 to $71.2 \%$ with a mean of $32.6 \%$.
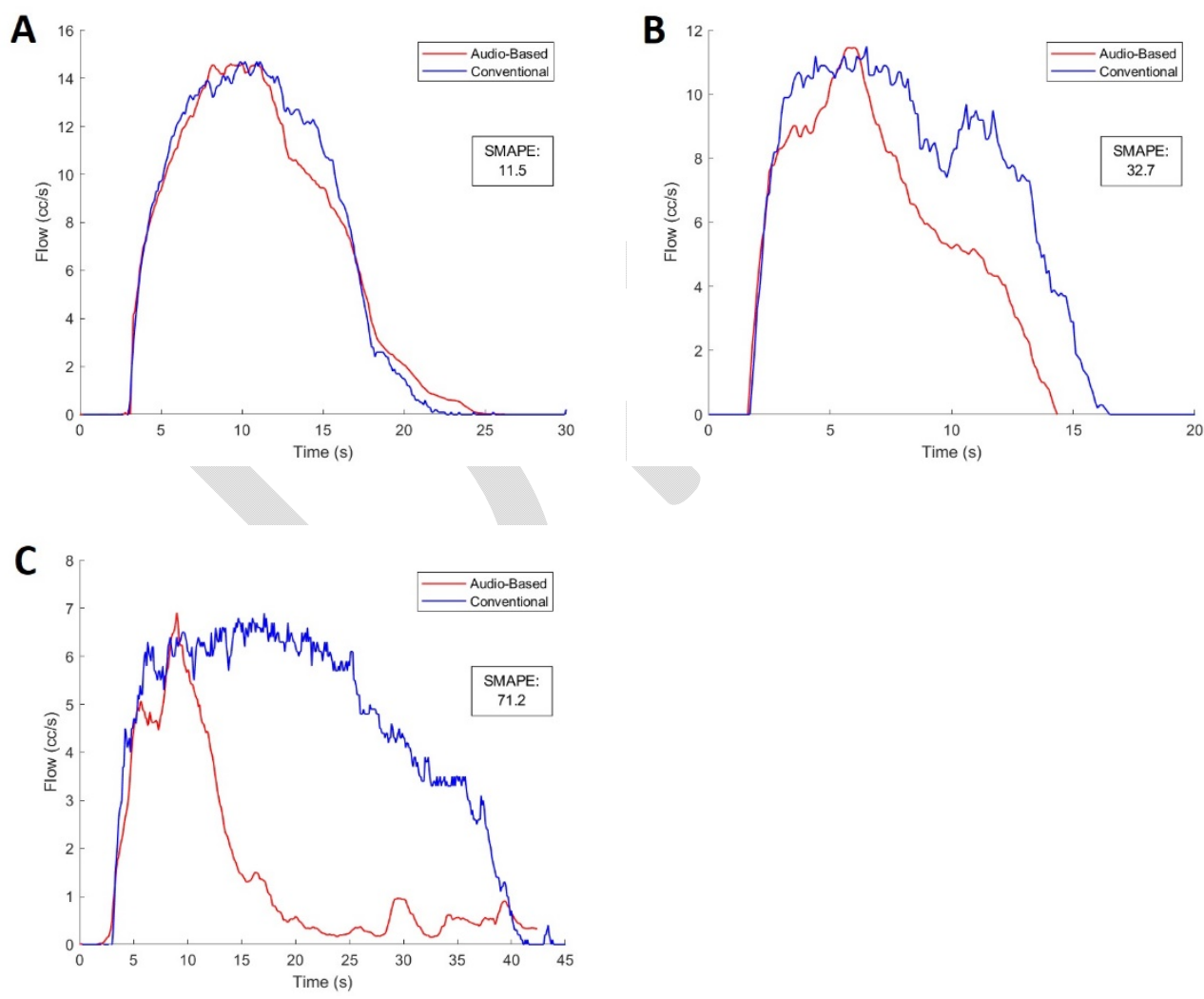


\begin{tabular}{|c|c|}
\hline Number of patients & 44 \\
\hline Age $(y)$, mean $(\mathrm{SD})$ & $59.7(14.1)$ \\
\hline \multicolumn{2}{|c|}{ Reason for clinic visit, no. (\%) } \\
\hline Hematuria & $5(11)$ \\
\hline LUTS & $13(30)$ \\
\hline Post-renal transplant & $14(32)$ \\
\hline Surveillance cystoscopy & $12(27)$ \\
\hline \multicolumn{2}{|c|}{ Lower urinary tract diagnoses, no. (\%) } \\
\hline $\mathrm{BPH}$ & $15(34)$ \\
\hline Bladder stones & $1(2)$ \\
\hline Overactive bladder & $1(2)$ \\
\hline Urethral stricture & $2(5)$ \\
\hline Urothelial carcinoma & $12(27)$ \\
\hline
\end{tabular}

BPH: benign prostatic hyperplasia; LUTS: lower urinary tract symptoms; SD: standard deviation. 